\title{
Heat flow, Brownian motion and Newtonian capacity
}

\author{
M. van den Berg \\ School of Mathematics, University of Bristol, University Walk, Bristol BS8 1TW, United Kingdom
}

Received 15 January 2006; accepted 1 March 2006

Available online 13 December 2006

\section{Abstract}

Let $K$ be a compact, non-polar set in $\mathbb{R}^{m}(m \geqslant 3)$ and let $u$ be the unique weak solution of $\Delta u=\frac{\partial u}{\partial t}$ on $\mathbb{R}^{m} \backslash K \times(0, \infty)$, $u(x ; 0)=0$ on $\mathbb{R}^{m} \backslash K$ and $u(x ; t)=1$ for all $x$ on the boundary of $K$ and for all $t>0$. The asymptotic behaviour of $u(x ; t)$ as $t$ tends to infinity is obtained up to order $\mathrm{O}\left(t^{-m / 2}\right)$.

๑) 2006 Elsevier Masson SAS. All rights reserved.

\section{Résumé}

Soit $K$ un ensemble compact, non-polaire dans $\mathbb{R}^{m}(m \geqslant 3)$ et soit $u$ l'unique solution faible de $\Delta u=\frac{\partial u}{\partial t}$ sur $\mathbb{R}^{m} \backslash K \times(0, \infty)$, $u(x ; 0)=0$ sur $\mathbb{R}^{m} \backslash K$ et $u(x ; t)=1$ pour tout $x$ sur la frontière de $K$ et tout $t>0$. On obtient le comportement asymptotique de $u(x, t)$ quand $t$ tend vers l'infini avec un reste $\mathrm{O}\left(t^{-m / 2}\right)$.

(c) 2006 Elsevier Masson SAS. All rights reserved.

MSC: 35K20; 60J65; 60J45

Keywords: Heat flow; Brownian motion; Newtonian capacity

\section{Introduction}

Let $K$ be a compact, non-polar set in Euclidean space $\mathbb{R}^{m}(m \geqslant 3)$ with boundary $\partial K$ and let $u: \mathbb{R}^{m} \backslash K \times[0, \infty) \rightarrow$ $\mathbb{R}$ be the unique weak solution of

$$
\Delta u=\frac{\partial u}{\partial t}, \quad x \in \mathbb{R}^{m} \backslash K, t>0,
$$

with boundary condition

$$
u(x ; t)=1, \quad x \in \partial K, t>0,
$$

and initial condition

$$
u(x ; 0)=0, \quad x \in \mathbb{R}^{m} \backslash K .
$$

It is well known that

$$
\lim _{t \rightarrow \infty} u(x ; t)=h_{K}(x), \quad x \in \mathbb{R}^{m} \backslash K,
$$

E-mail address: M.vandenBerg@bris.ac.uk (M. van den Berg). 
where $h_{K}$ is the unique function which is harmonic on $\mathbb{R}^{m} \backslash K$, which equals 1 on the regular points of $K$, and which vanishes at infinity.

S.C. Port [8], [10, pp. 64, 65] proved that if $K$ is a compact and non-polar set in $\mathbb{R}^{m}(m \geqslant 3)$ then for $t \rightarrow \infty$

$$
u(x ; t)=h_{K}(x)-\left(\frac{m}{2}-1\right)^{-1}(4 \pi)^{-m / 2} C(K)\left(1-h_{K}(x)\right) t^{(2-m) / 2}+\mathrm{o}\left(t^{(2-m) / 2}\right),
$$

where $C(K)$ is the Newtonian capacity of $K$.

Formula (5) was first proved by A. Joffe [7] in the special case where $m=3$ and where $K$ has positive Lebesgue measure $|K|$. Subsequently F. Spitzer [12, p. 114] proved formula (5) for arbitrary compact, non-polar sets in $\mathbb{R}^{3}$ and obtained the asymptotic behaviour of the total amount of heat $E_{K}(t)$ in $\mathbb{R}^{m} \backslash K$ at time $t$ defined by

$$
E_{K}(t)=\int_{\mathbb{R}^{m} \backslash K} u(x ; t) \mathrm{d} x .
$$

He showed that for $m=3$ and $t \rightarrow \infty$

$$
E_{K}(t)=C(K) t+\frac{1}{2 \pi^{3 / 2}} C(K)^{2} t^{1 / 2}+\mathrm{o}\left(t^{1 / 2}\right) .
$$

J.-F. Le Gall [4-6] and Port [11] obtained refinements of (7) and extensions to $m \geqslant 4$ and $m=2$ without the use of (5). Port also obtained the large $t$ behaviour of $u$ in the case where $K$ is a non-polar compact set in $\mathbb{R}^{2}$ [9].

The main result of this paper concerns the analysis of the remainder estimate $\mathrm{o}\left(t^{(2-m) / 2}\right)$ in (5). For $m \geqslant 5$ we show that this remainder can be improved to $\mathrm{O}\left(t^{-m / 2}\right)$. A new term of order $(\log t) / t^{2}$ shows up for $m=4$ before we recover the remainder $\mathrm{O}\left(t^{-2}\right)$. A remarkable cancellation of two terms of order $t^{-1}$ and four terms of order $(\log t) / t^{3 / 2}$ takes place for $m=3$, resulting in the sharp remainder $\mathrm{O}\left(t^{-3 / 2}\right)$.

Theorem 1. Let $K$ be a compact and non-polar set in $\mathbb{R}^{m}$.

(i) If $m=3,5,6, \ldots$ then for $x \in \mathbb{R}^{m} \backslash K$ and $t \rightarrow \infty$

$$
u(x ; t)=h_{K}(x)-\left(\frac{m}{2}-1\right)^{-1}(4 \pi)^{-m / 2} C(K)\left(1-h_{K}(x)\right) t^{(2-m) / 2}+\mathrm{O}\left(t^{-m / 2}\right) .
$$

(ii) If $m=4$ then for $x \in \mathbb{R}^{4} \backslash K$ and $t \rightarrow \infty$

$$
u(x ; t)=h_{K}(x)-(4 \pi)^{-2} C(K)\left(1-h_{K}(x)\right) t^{-1}+2(4 \pi)^{-4} C(K)^{2}\left(1-h_{K}(x)\right) \frac{\log t}{t^{2}}+\mathrm{O}\left(t^{-2}\right) .
$$

(iii) The remainder in (8) is sharp for a ball in $\mathbb{R}^{3}$.

(iv) The remainder $\mathrm{O}\left(t^{-m / 2}\right)$ in (8) and (9) is uniform in $x$ on compact subsets of $\mathbb{R}^{m} \backslash K$.

The results described in Theorem 1 have an equivalent probabilistic formulation. Let $\left(B(s), s \geqslant 0 ; \mathbb{P}_{x}, x \in \mathbb{R}^{m}\right)$ be a Brownian motion with generator $\Delta$. For $x \in \mathbb{R}^{m}$ we define the first hitting time of $K$ by

$$
T_{K}=\inf \{s \geqslant 0: B(s) \in K\},
$$

and $T_{K}=+\infty$ if the infimum is taken over the empty set. It is a classical result that

$$
u(x ; t)=\mathbb{P}_{x}\left[T_{K}<t\right], \quad x \in \mathbb{R}^{m}, t>0,
$$

where we have extended both $u$ and $h_{K}$ to all of $\mathbb{R}^{m}$ by putting $u \equiv h_{K} \equiv 1$ on $K$. For $x \in \mathbb{R}^{m}(m \geqslant 3)$ we define the last exit time of $K$ by

$$
L_{K}=\sup \{s \geqslant 0: B(s) \in K\},
$$

and $L_{K}=+\infty$ if the supremum is taken over the empty set. The law of $L_{K}$ is given by [10, p. 61]

$$
\mathbb{P}_{x}\left[L_{K}<t\right]=\int_{0}^{t} \mathrm{~d} s \int \mu_{K}(\mathrm{~d} y) p(x, y ; s),
$$


where

$$
p(x, y ; s)=(4 \pi s)^{-m / 2} \mathrm{e}^{-|x-y|^{2} /(4 s)},
$$

and where $\mu_{K}$ is the equilibrium measure supported on $K$ with

$$
\int \mu_{K}(\mathrm{~d} y)=C(K)
$$

It follows that

$$
h_{K}(x)=\mathbb{P}_{x}\left[T_{K}<\infty\right]=\mathbb{P}_{x}\left[L_{K}<\infty\right]=c_{m} \int \mu_{K}(\mathrm{~d} y)|x-y|^{2-m},
$$

where

$$
c_{m}=4^{-1} \pi^{-m / 2} \Gamma((m-2) / 2) .
$$

Since

$$
\mathbb{P}_{x}\left[t<L_{K}<\infty\right]=\int_{t}^{\infty} \mathrm{d} s \int \mu_{K}(\mathrm{~d} y) p(x, y ; s),
$$

and

$$
(4 \pi s)^{-m / 2}\left(1-|x-y|^{2} /(4 s)\right) \leqslant p(x, y ; s) \leqslant(4 \pi s)^{-m / 2},
$$

we have that

$$
\mathbb{P}_{x}\left[t<L_{K}<\infty\right]=\left(\frac{m}{2}-1\right)^{-1}(4 \pi)^{-m / 2} C(K) t^{(2-m) / 2}+\mathrm{O}\left(t^{-m / 2}\right) .
$$

Using (11), (16) and (20) we can rewrite (8), (9) as follows.

Proposition 2. Let $K$ be a compact and non-polar set in $\mathbb{R}^{m}$.

(i) If $m=3,5,6, \ldots$ then for $x \in \mathbb{R}^{m} \backslash K$ and $t \rightarrow \infty$

$$
\mathbb{P}_{x}\left[t<T_{K}<\infty\right]=\mathbb{P}_{x}\left[T_{K}=\infty\right] \mathbb{P}_{x}\left[t<L_{K}<\infty\right]+\mathrm{O}\left(t^{-m / 2}\right) .
$$

(ii) If $m=4$ then for $x \in \mathbb{R}^{4} \backslash K$ and $t \rightarrow \infty$

$$
\mathbb{P}_{x}\left[t<T_{K}<\infty\right]=\mathbb{P}_{x}\left[T_{K}=\infty\right] \mathbb{P}_{x}\left[t<L_{K}<\infty\right]-2(4 \pi)^{-4} C(K)^{2} \mathbb{P}_{x}\left[T_{K}=\infty\right] \frac{\log t}{t^{2}}+\mathrm{O}\left(t^{-2}\right)
$$

It is well known [4, p. 392] that if $m=3$ and $K=B(0 ; R)$ (the closed ball with center 0 and radius $R$ ) then for $|x| \geqslant R$

$$
\mathbb{P}_{x}\left[t<T_{B(0 ; R)}<\infty\right]=\int_{t}^{\infty} \mathrm{d} s\left(4 \pi s^{3}\right)^{-1 / 2} \frac{R(|x|-R)}{|x|} \mathrm{e}^{-(|x|-R)^{2} /(4 s)} .
$$

Moreover for a ball $B(0 ; R)$ in $\mathbb{R}^{3}$ the corresponding equilibrium measure is concentrated on $\partial B(0 ; R)$ and proportional to the surface measure, with constant of proportionality equal to $R^{-1}$. This gives by (18)

$$
\mathbb{P}_{x}\left[T_{B(0 ; R)}=\infty\right]=\frac{|x|-R}{|x|},
$$

and

$$
\mathbb{P}_{x}\left[t<L_{B(0 ; R)}<\infty\right]=\int_{t}^{\infty} \mathrm{d} s(4 \pi s)^{-1 / 2}|x|^{-1}\left(1-\mathrm{e}^{-|x| R / s}\right) \mathrm{e}^{-(|x|-R)^{2} /(4 s)} .
$$


It is a straightforward computation to show that, by (23)-(25), for $m=3$

$$
\begin{aligned}
\mathbb{P}_{x}\left[t<T_{B(0 ; R)}<\infty\right]= & \mathbb{P}_{x}\left[T_{B(0 ; R)}=\infty\right] \mathbb{P}_{x}\left[t<L_{B(0 ; R)}<\infty\right] \\
& +\frac{1}{6 \pi^{1 / 2}} \mathbb{P}_{x}\left[T_{B(0 ; R)}=\infty\right]|x| R^{2} t^{-3 / 2}+\mathrm{O}\left(t^{-5 / 2}\right) .
\end{aligned}
$$

This proves the assertion in Theorem 1(iii).

The main stratagem which permeates the proof of Proposition 2 is to replace $T_{K}$ by $L_{K}$ at "every possible opportunity" and to use the strong Markov property to control terms like $\mathbb{P}_{x}\left[T_{K}<t<L_{K}\right]$. For a different application of these techniques we refer to the study of the expected volume of a Wiener sausage in $\mathbb{R}^{3}$ associated to the compact set $K$ [4]. There Spitzer's formula (7) was improved up to order $\mathrm{O}\left(t^{-1 / 2}\right)$ proving a conjecture by M. Kac. See $[1-3,13]$ for more recent applications.

It turns out that a single application of the strong Markov property (Proposition 4) supplemented by additional estimates (Lemma 3) is sufficient to prove Proposition 2 for $m \geqslant 5$. However, for $m=4$ or $m=3$ the strong Markov property has to be applied twice respectively six times (Propositions 5 and 8). The reason is that for $m=3$ two nontrivial terms of order $t^{-1}$ and four non-trivial terms of order $(\log t) / t^{3 / 2}$ contribute to $\mathbb{P}_{x}\left[t<T_{K}<\infty\right]$. Lengthy calculations using the above techniques finally result in the cancellation of these non-trivial terms. Such a cancellation does not take place for $m=4$, and this results in the $(\log t) / t^{2}$ contribution in (9).

The analysis of the $\mathrm{O}\left(t^{-m / 2}\right)$ remainder in Proposition 2 is complicated since the distribution of the random variable $B\left(T_{K}\right)$ on the regular part of $\partial K$ enters at each application of the strong Markov property. Unlike the special case of a ball in $\mathbb{R}^{3}$ we do not expect a simple improvement of the remainder.

This paper is organized as follows. In Section 2 we prove some basic estimates (Lemma 3) which will be used throughout the paper. Proposition 4 is the key estimate from which Proposition 2 follows for $m \geqslant 5$. In Section 3 we use Proposition 4 to obtain a further refinement (Proposition 5) from which Proposition 2 follows for $m=4$. Finally in Section 4 we complete the proof of Proposition 2 for $m=3$ by refining Proposition 5 (Proposition 8). The proof of Proposition 8 follows the same strategy as the proof of Proposition 5, and has been omitted.

\section{Proof of Proposition 2 for $m \geqslant 5$}

It is convenient to introduce some further notation. For $c \in \mathbb{R}^{m}$ and $K$ compact in $\mathbb{R}^{m}$ we define

$$
R(c)=\inf \{\rho \geqslant 0: K \subset B(c ; \rho)\},
$$

where $B(c ; \rho)$ is the closed ball with center $c$ and radius $\rho$. Let

$$
R=\inf \left\{R(c): c \in \mathbb{R}^{m}\right\} .
$$

The infima in (27) and (28) are attained and we may assume without loss of generality that the latter is attained at $c=0$.

Lemma 3. Let $K$ be a compact and non-polar set in $\mathbb{R}^{m}(m \geqslant 3)$. Then for $0<s<t<\infty$

$$
\begin{aligned}
\mathbb{P}_{x}\left[t<T_{K}<\infty\right] & \leqslant \mathbb{P}_{x}\left[t<L_{K}<\infty\right] \\
& \leqslant 1 \wedge\left(\frac{m}{2}-1\right)^{-1}(4 \pi)^{-m / 2} C(K) t^{(2-m) / 2}, \\
\mathbb{P}_{x}\left[s<L_{K}<t\right] & \leqslant 1 \wedge\left(\frac{m}{2}-1\right)^{-1}(4 \pi)^{-m / 2} C(K)\left(s^{(2-m) / 2}-t^{(2-m) / 2}\right),
\end{aligned}
$$

and for $z \in K$

$$
\left|\mathbb{P}_{x}\left[t<L_{K}<\infty\right]-\mathbb{P}_{z}\left[t<L_{K}<\infty\right]\right| \leqslant 1 \wedge C_{x, K} t^{-m / 2},
$$

where

$$
C_{x, K}=(|x|+R)(|x|+3 R) C(K) .
$$


For any Borel set $E$ of $[0, t]$

$$
\int_{E} \mathrm{~d} s \int \mu_{K}(\mathrm{~d} y) p(x, y ; t-s) \leqslant 1 .
$$

Let $T>0$ be arbitrary. There exists a constant $C$ depending on $T$ and on $K$ such that for all $t>T, 0<s<t$ and $x \in \mathbb{R}^{m}$

$$
\mathbb{P}_{x}\left[s<T_{K}<t\right] \leqslant C\left(T(t-T)^{-m / 2} \vee(t-s) s^{-m / 2}\right) .
$$

Proof. Estimate (29) follows immediately from the fact that $L_{K} \geqslant T_{K}$ and (18), (19).

Estimate (30) follows from

$$
\mathbb{P}_{x}\left[s<L_{K}<t\right]=\int_{s}^{t} \mathrm{~d} \tau \int \mu_{K}(\mathrm{~d} y) p(x, y ; \tau),
$$

and the bound in the right-hand side of (19).

To prove (31) we note that by (18)

$$
\begin{aligned}
\left|\mathbb{P}_{x}\left[t<L_{K}<\infty\right]-\mathbb{P}_{z}\left[t<L_{K}<\infty\right]\right| & \leqslant \int_{t}^{\infty} \mathrm{d} s(4 \pi s)^{-m / 2} \int \mu_{K}(\mathrm{~d} y)\left|\mathrm{e}^{-|x-y|^{2} /(4 s)}-\mathrm{e}^{-|z-y|^{2} /(4 s)}\right| \\
& \leqslant \int_{t}^{\infty} \mathrm{d} s(4 \pi s)^{-m / 2}(4 s)^{-1} \int \mu_{K}(\mathrm{~d} y)|| x-\left.y\right|^{2}-|z-y|^{2} \mid \\
& \leqslant t^{-m / 2} \int \mu_{K}(\mathrm{~d} y)(|x|+|z|)(|x|+|z|+2|y|) \\
& \leqslant C_{x, K} t^{-m / 2}
\end{aligned}
$$

since both $y$ and $z \in K \subset B(0 ; R)$.

Since $p$ is non-negative

$$
\begin{aligned}
\int_{E} \mathrm{~d} s \int \mu_{K}(\mathrm{~d} y) p(x, y ; t-s) & \leqslant \int_{[0, t]} \mathrm{d} s \int \mu_{K}(\mathrm{~d} y) p(x, y ; t-s) \\
& =\mathbb{P}_{x}\left[L_{K}<t\right] \leqslant 1 .
\end{aligned}
$$

This proves (33).

The proof of (34) relies on the following [4,11,12]. For $m \geqslant 3$

$$
\int_{\mathbb{R}^{m}} \mathrm{~d} y \mathbb{P}_{y}\left[T_{K}<t\right]=C(K) t+\mathrm{o}(t), \quad t \rightarrow \infty .
$$

Hence there exists $T_{1}$ such that for all $t \geqslant T_{1}$

$$
\int_{\mathbb{R}^{m}} \mathrm{~d} y \mathbb{P}_{y}\left[T_{K}<t\right] \leqslant 2 C(K) t .
$$

By the Markov property at time $s$ we have that

$$
\mathbb{P}_{x}\left[s<T_{K}<t\right]=\int_{\mathbb{R}^{m}} \mathrm{~d} y p_{\mathbb{R}^{m} \backslash K}(x, y ; s) \mathbb{P}_{y}\left[T_{K}<t-s\right],
$$

where $p_{\mathbb{R}^{m} \backslash K}(\cdot, \cdot ; \cdot)$ is the Dirichlet heat kernel for the open set $\mathbb{R}^{m} \backslash K$ (i.e. the transition density of Brownian motion with killing on $K$ ). By domain monotonicity of the Dirichlet heat kernel

$$
p_{\mathbb{R}^{m} \backslash K}(x, y ; s) \leqslant p(x, y ; s) \leqslant(4 \pi s)^{-m / 2} .
$$


We first consider the case $t-s>T_{1}$. Then by (39)-(41)

$$
\begin{aligned}
\mathbb{P}_{x}\left[s<T_{K}<t\right] & \leqslant(4 \pi s)^{-m / 2} \int_{\mathbb{R}^{m}} \mathrm{~d} y \mathbb{P}_{y}\left[T_{K}<t-s\right] \\
& \leqslant 2(4 \pi s)^{-m / 2} C(K)(t-s) .
\end{aligned}
$$

Next suppose that $T<T_{1}$ and $t-s \in\left[T, T_{1}\right]$. Then by monotonicity

$$
\begin{aligned}
\int_{\mathbb{R}^{m}} \mathrm{~d} y \mathbb{P}_{y}\left[T_{K}<t-s\right] & \leqslant \int_{\mathbb{R}^{m}} \mathrm{~d} y \mathbb{P}_{y}\left[T_{K}<T_{1}\right] \\
& \leqslant 2 C(K) T_{1} \leqslant 2 C(K) \frac{T_{1}}{T}(t-s),
\end{aligned}
$$

and

$$
\mathbb{P}_{x}\left[s<T_{K}<t\right] \leqslant 2(4 \pi s)^{-m / 2} C(K) \frac{T_{1}}{T}(t-s) .
$$

Combining (42) and (44) we obtain that

$$
\mathbb{P}_{x}\left[s<T_{K}<t\right] \leqslant C s^{-m / 2}(t-s), \quad t-s \geqslant T,
$$

with $C$ given by

$$
C=2(4 \pi)^{-m / 2} C(K)\left(1 \vee \frac{T_{1}}{T}\right) .
$$

By (45)

$$
\mathbb{P}_{x}\left[s<T_{K}<t\right] \leqslant \mathbb{P}_{x}\left[t-T<T_{K}<t\right] \leqslant C T(t-T)^{-m / 2}, \quad t-s \leqslant T,
$$

and (34) follows from (45)-(47).

Proposition 4. Let $K$ be a compact and non-polar set in $\mathbb{R}^{m}(m \geqslant 3)$. Then for $t \rightarrow \infty$

$$
\begin{aligned}
\mathbb{P}_{x}\left[t<T_{K}<\infty\right]= & \mathbb{P}_{x}\left[T_{K}=\infty\right] \mathbb{P}_{x}\left[t<L_{K}<\infty\right]+\mathbb{P}_{x}\left[t<T_{K}<\infty\right] \mathbb{P}_{x}\left[t<L_{K}<\infty\right] \\
& -\int_{0}^{t} \mathrm{~d} s \mathbb{P}_{x}\left[s<T_{K}<t\right] \int \mu_{K}(\mathrm{~d} y) p(x, y ; t-s)+\mathrm{O}\left(t^{-m / 2}\right) .
\end{aligned}
$$

Proof. Note that

$$
\mathbb{P}_{x}\left[t<T_{K}<\infty\right]=\mathbb{P}_{x}\left[t<L_{K}<\infty\right]-\mathbb{P}_{x}\left[T_{K}<t<L_{K}\right] .
$$

By the strong Markov property

$$
\mathbb{P}_{x}\left[T_{K}<t<L_{K}\right]=E_{x}\left\{\int_{0}^{t} 1_{T_{K} \in \mathrm{d} s} \mathbb{P}_{B\left(T_{K}\right)}\left[t-s<L_{K}<\infty\right]\right\} .
$$

Using Lemma 3, (31) with $z=B\left(T_{K}\right)$

$$
\left|\mathbb{P}_{B\left(T_{K}\right)}\left[t-s<L_{K}<\infty\right]-\mathbb{P}_{x}\left[t-s<L_{K}<\infty\right]\right| \leqslant 1 \wedge C_{x, K}(t-s)^{-m / 2} .
$$

If we can show that

$$
E_{x}\left\{\int_{0}^{t} 1_{T_{K} \in \mathrm{d} s}\left(1 \wedge C_{x, K}(t-s)^{-m / 2}\right)\right\}=\mathrm{O}\left(t^{-m / 2}\right),
$$

then, by (50)-(52), 


$$
\begin{aligned}
\mathbb{P}_{x}\left[T_{K}<t<L_{K}\right]= & E_{x}\left\{\int_{0}^{t} 1_{T_{K} \in \mathrm{d} s} \mathbb{P}_{x}\left[t-s<L_{K}<\infty\right]\right\}+\mathrm{O}\left(t^{-m / 2}\right) \\
= & \int_{0}^{t} \mathrm{~d} s \frac{\mathrm{d}}{\mathrm{d} s}\left(\mathbb{P}_{x}\left[T_{K}<s\right]-\mathbb{P}_{x}\left[T_{K}<t\right]\right) \mathbb{P}_{x}\left[t-s<L_{K}<\infty\right]+\mathrm{O}\left(t^{-m / 2}\right) \\
= & \mathbb{P}_{x}\left[T_{K}<t\right] \mathbb{P}_{x}\left[t<L_{K}<\infty\right] \\
& +\int_{0}^{t} \mathrm{~d} s \mathbb{P}_{x}\left[s<T_{K}<t\right] \frac{\mathrm{d}}{\mathrm{d} s} \mathbb{P}_{x}\left[t-s<L_{K}<\infty\right]+\mathrm{O}\left(t^{-m / 2}\right) .
\end{aligned}
$$

This implies Proposition 4 since, by (18),

$$
\frac{\mathrm{d}}{\mathrm{d} s} \mathbb{P}_{x}\left[t-s<L_{K}<\infty\right]=\int \mu_{K}(\mathrm{~d} y) p(x, y ; t-s) .
$$

To prove (52) we note that

$$
\begin{aligned}
& E_{x}\left\{\int_{0}^{t} 1_{T_{K} \in \mathrm{d} s}\left(1 \wedge C_{x, K}(t-s)^{-m / 2}\right)\right\} \\
& \quad=\int_{0}^{t} \mathrm{~d} s \frac{\mathrm{d}}{\mathrm{d} s}\left(\mathbb{P}_{x}\left[T_{K}<s\right]-\mathbb{P}_{x}\left[T_{K}<t\right]\right)\left(1 \wedge C_{x, K}(t-s)^{-m / 2}\right) \\
& \quad=\mathbb{P}_{x}\left[T_{K}<t\right]\left(1 \wedge C_{x, K} t^{-m / 2}\right)+\frac{m}{2} C_{x, K} \int_{0}^{t^{*}} \mathrm{~d} s \mathbb{P}_{x}\left[s<T_{K}<t\right](t-s)^{-(m+2) / 2},
\end{aligned}
$$

where

$$
t^{*}=(t-T) \vee 0,
$$

and

$$
T=C_{x, K}^{2 / m}
$$

The first term in the right-hand side of (55) is $\mathrm{O}\left(t^{-m / 2}\right)$. To estimate the second term in the right-hand side of (55) we suppose that $t>T$ and use Lemma 3 with $T=C_{x, K}^{2 / m}$ to obtain that

$$
\begin{aligned}
& \int_{0}^{t^{*}} \mathrm{~d} s \mathbb{P}_{x}\left[s<T_{K}<t\right](t-s)^{-(m+2) / 2} \\
& \leqslant \int_{0}^{(t-T) / 2} \mathrm{~d} s(t-s)^{-(m+2) / 2}+\int_{(t-T) / 2}^{t-T} \mathrm{~d} s C s^{-m / 2}(t-s)^{-m / 2} \\
& \leqslant((t+T) / 2)^{-m / 2}+C((t-T) / 2)^{-m / 2} \int_{0}^{t-T} \mathrm{~d} s(t-s)^{-m / 2}=\mathrm{O}\left(t^{-m / 2}\right) .
\end{aligned}
$$

We conclude this section with the proof of Proposition 2 for $m \geqslant 5$. By Lemma 3, (29), the second term in the right-hand side of (48) is $\mathrm{O}\left(t^{2-m}\right)$ and hence is $\mathrm{O}\left(t^{-m / 2}\right)$ for $m \geqslant 4$. By (19) and (15) we have for any $z \in \mathbb{R}^{m}$

$$
\int \mu_{K}(\mathrm{~d} y) p(z, y ; t-s) \leqslant C(K) t^{-m / 2}, \quad s \in[0, t / 2] .
$$


Hence, by (29) and (59), we have for $m \geqslant 5$

$$
\int_{0}^{t / 2} \mathrm{~d} s \mathbb{P}_{x}\left[s<T_{K}<t\right] \int \mu_{K}(\mathrm{~d} y) p(x, y ; t-s) \leqslant C(K) t^{-m / 2} \int_{0}^{t / 2} \mathrm{~d} s\left(1 \wedge C(K) s^{1-m / 2}\right)=\mathrm{O}\left(t^{-m / 2}\right) .
$$

By (34) we have for $m \geqslant 5$

$$
\int_{t / 2}^{t-T} \mathrm{~d} s \mathbb{P}_{x}\left[s<T_{K}<t\right] \int \mu_{K}(\mathrm{~d} y) p(x, y ; t-s) \leqslant C \int_{t / 2}^{t-T} \mathrm{~d} s s^{-m / 2} C(K)(t-s)^{1-m / 2}=\mathrm{O}\left(t^{-m / 2}\right) .
$$

By (34) and (33) for $E=[t-T, t]$ we have

$$
\int_{t-T}^{t} \mathrm{~d} s \mathbb{P}_{x}\left[s<T_{K}<t\right] \int \mu_{K}(\mathrm{~d} y) p(x, y ; t-s) \leqslant C T(t-T)^{-m / 2}=\mathrm{O}\left(t^{-m / 2}\right) .
$$

By (60)-(62) and Proposition 4 we conclude that (21) holds for $m \geqslant 5$.

\section{Proof of Proposition 2 for $m=4$}

The proof of Proposition 2 for $m=4$ and $m=3$ relies on the asymptotic analysis of the third term in the right-hand side of (48).

Proposition 5. Let $K$ be a compact and non-polar set in $\mathbb{R}^{m}(m \geqslant 3)$. Then for $t \rightarrow \infty$

$$
\begin{aligned}
& \int_{0}^{t} \mathrm{~d} s \mathbb{P}_{x}\left[s<T_{K}<t\right] \int \mu_{K}(\mathrm{~d} y) p(x, y ; t-s) \\
& \quad=\mathbb{P}_{x}\left[T_{K}=\infty\right] \int_{0}^{t} \mathrm{~d} s \mathbb{P}_{x}\left[s<L_{K}<t\right] \int \mu_{K}(\mathrm{~d} y) p(x, y ; t-s)+\sum_{i=1}^{4} A_{i}+\mathrm{O}\left(t^{-m / 2}\right),
\end{aligned}
$$

where

$$
\begin{aligned}
A_{1}= & \int_{0}^{t} \mathrm{~d} s \mathbb{P}_{x}\left[s<T_{K}<t\right] \mathbb{P}_{x}\left[t-s<L_{K}<\infty\right] \int \mu_{K}(\mathrm{~d} y) p(x, y ; t-s), \\
A_{2}= & \int_{0}^{t} \mathrm{~d} s \mathbb{P}_{x}\left[s<T_{K}<\infty\right] \mathbb{P}_{x}\left[s<L_{K}<t\right] \int \mu_{K}(\mathrm{~d} y) p(x, y ; t-s), \\
A_{3}= & \int_{0}^{t} \mathrm{~d} s \int_{s}^{t} \mathrm{~d} \tau \mathbb{P}_{x}\left[\tau<T_{K}<t\right] \int \mu_{K}(\mathrm{~d} z) p(x, z ; t-\tau) \int \mu_{K}(\mathrm{~d} y) p(x, y ; t-s), \\
A_{4}= & \int_{0}^{t} \mathrm{~d} s \int_{0}^{s} \mathrm{~d} \tau \mathbb{P}_{x}\left[\tau<T_{K}<s\right] \int \mu_{K}(\mathrm{~d} z)(p(x, z ; t-\tau)-p(x, z ; s-\tau)) \int \mu_{K}(\mathrm{~d} y) p(x, y ; t-s) .
\end{aligned}
$$

Proof. Since

$$
\mathbb{P}_{x}\left[s<T_{K}<t\right]=\mathbb{P}_{x}\left[s<L_{K}<t\right]+\mathbb{P}_{x}\left[T_{K}<t<L_{K}\right]-\mathbb{P}_{x}\left[T_{K}<s<L_{K}\right],
$$

we have that the left-hand side of (63) equals 


$$
\begin{aligned}
& \int_{0}^{t} \mathrm{~d} s \mathbb{P}_{x}\left[s<L_{K}<t\right] \int \mu_{K}(\mathrm{~d} y) p(x, y ; t-s) \\
& \quad+\int_{0}^{t} \mathrm{~d} s\left(\mathbb{P}_{x}\left[T_{K}<t<L_{K}\right]-\mathbb{P}_{x}\left[T_{K}<s<L_{K}\right]\right) \int \mu_{K}(\mathrm{~d} y) p(x, y ; t-s) .
\end{aligned}
$$

By the strong Markov property we can write the second term in (69) as

$$
\begin{aligned}
& \int_{0}^{t} \mathrm{~d} s E_{x}\left\{\int_{0}^{t} 1_{T_{K} \in \mathrm{d} \tau} \mathbb{P}_{B\left(T_{K}\right)}\left[t-\tau<L_{K}<\infty\right]\right. \\
& \left.-\int_{0}^{s} 1_{T_{K} \in \mathrm{d} \tau} \mathbb{P}_{B\left(T_{K}\right)}\left[s-\tau<L_{K}<\infty\right]\right\} \int \mu_{K}(\mathrm{~d} y) p(x, y ; t-s) .
\end{aligned}
$$

First we show that we can replace $B\left(T_{K}\right)$ in (70) by $x$ at a cost $\mathrm{O}\left(t^{-m / 2}\right)$. By (52)

$$
\begin{aligned}
& \int_{0}^{t} \mathrm{~d} s E_{x}\left\{\int_{0}^{t} 1_{T_{K} \in \mathrm{d} \tau}\left(1 \wedge C_{x, K}(t-\tau)^{-m / 2}\right)\right\} \int \mu_{K}(\mathrm{~d} y) p(x, y ; t-s) \\
& \leqslant E_{x}\left\{\int_{0}^{t} 1_{T_{K} \in \mathrm{d} \tau}\left(1 \wedge C_{x, K}(t-\tau)^{-m / 2}\right)\right\}=\mathrm{O}\left(t^{-m / 2}\right) .
\end{aligned}
$$

Moreover by (55)

$$
\begin{aligned}
& \int_{0}^{t} \mathrm{~d} s E_{x}\left\{\int_{0}^{s} 1_{T_{K} \in \mathrm{d} \tau}\left(1 \wedge C_{x, K}(s-\tau)^{-m / 2}\right)\right\} \int \mu_{K}(\mathrm{~d} y) p(x, y ; t-s) \\
& =\int_{0}^{t} \mathrm{~d} s \mathbb{P}_{x}\left[T_{K}<s\right]\left(1 \wedge C_{x, K} s^{-m / 2}\right) \int \mu_{K}(\mathrm{~d} y) p(x, y ; t-s) \\
& \quad+\int_{0}^{t} \mathrm{~d} s \int_{0}^{s^{*}} \mathrm{~d} \tau \mathbb{P}_{x}\left[\tau<T_{K}<s\right] C_{x, K} \frac{m}{2}(s-\tau)^{-(m+2) / 2} \int \mu_{K}(\mathrm{~d} y) p(x, y ; t-s),
\end{aligned}
$$

where

$$
s^{*}=(s-T) \vee 0 .
$$

By (59)

$$
\begin{gathered}
\int_{0}^{t / 2} \mathrm{~d} s \mathbb{P}_{x}\left[T_{K}<s\right]\left(1 \wedge C_{x, K} s^{-m / 2}\right) \int \mu_{K}(\mathrm{~d} y) p(x, y ; t-s) \leqslant C(K) t^{-m / 2}\left(\int_{0}^{\infty} \mathrm{d} s\left(1 \wedge C_{x, K} s^{-m / 2}\right)\right) \\
=\mathrm{O}\left(t^{-m / 2}\right) .
\end{gathered}
$$

By (33) with $E=[t / 2, t]$ 


$$
\begin{gathered}
\int_{t / 2}^{t} \mathrm{~d} s \mathbb{P}_{x}\left[T_{K}<s\right]\left(1 \wedge C_{x, K} s^{-m / 2}\right) \int \mu_{K}(\mathrm{~d} y) p(x, y ; t-s) \\
=C_{x, K}(t / 2)^{-m / 2} \int_{E} \mathrm{~d} s \int \mu_{K}(\mathrm{~d} y) p(x, y ; t-s) \\
=\mathrm{O}\left(t^{-m / 2}\right) .
\end{gathered}
$$

To estimate the second term in the right-hand side of (72) we have that the contribution from $s \in[T, 2 T]$ is bounded by

$$
\begin{aligned}
\int_{T}^{2 T} \mathrm{~d} s \int_{0}^{s-T} \mathrm{~d} \tau C_{x, K} \frac{m}{2}(s-\tau)^{-(m+2) / 2} \int \mu_{K}(\mathrm{~d} y) p(x, y ; t-s) & \leqslant \int_{T}^{2 T} \mathrm{~d} s \int \mu_{K}(\mathrm{~d} y) p(x, y ; t-s) \\
& \leqslant C(K) T(t-2 T)^{-m / 2} .
\end{aligned}
$$

The interval $[2 T, t / 2]$ contributes at most, by (34) and (59),

$$
\begin{aligned}
& \int_{2 T}^{t / 2} \mathrm{~d} s \int_{0}^{(s-T) / 2} \mathrm{~d} \tau C_{x, K} \frac{m}{2}(s-\tau)^{-(m+2) / 2} \int \mu_{K}(\mathrm{~d} y) p(x, y ; t-s) \\
& \quad+\int_{2 T}^{t / 2} \mathrm{~d} s \int_{(s-T) / 2}^{s-T} \mathrm{~d} \tau C C_{x, K} \frac{m}{2} \tau^{-m / 2}(s-\tau)^{-m / 2} \int \mu_{K}(\mathrm{~d} y) p(x, y ; t-s) \\
& \leqslant C(K) C_{x, K} t^{-m / 2} \int_{2 T}^{t / 2} \mathrm{~d} s \int_{-\infty}^{(s-T) / 2} \mathrm{~d} \tau \frac{m}{2}(s-\tau)^{-(m+2) / 2} \\
& \quad+C C(K) C_{x, K} t^{-m / 2} \int_{2 T}^{t / 2} \mathrm{~d} s((s-T) / 2)^{-m / 2} \int_{-\infty}^{s-T} \mathrm{~d} \tau \frac{m}{2}(s-\tau)^{-m / 2} \\
& =\mathrm{O}\left(t^{-m / 2}\right) .
\end{aligned}
$$

The interval $[t / 2, t]$ contributes at most, by (33) and (34),

$$
\begin{aligned}
& \sup _{t / 2<s<t}\left\{\int_{0}^{(s-T) / 2} \mathrm{~d} \tau C_{x, K} \frac{m}{2}(s-\tau)^{-(m+2) / 2}+\int_{(s-T) / 2}^{s-T} \mathrm{~d} \tau C C_{x, K} \frac{m}{2} \tau^{-m / 2}(s-\tau)^{-m / 2}\right\} \\
& \leqslant \sup _{t / 2<s<t}\left\{C_{x, K}((s+T) / 2)^{-m / 2}+3 C C_{x, K}((s-T) / 2)^{-m / 2} T^{(2-m) / 2}\right\}=\mathrm{O}\left(t^{-m / 2}\right) .
\end{aligned}
$$

By (74)-(78) we conclude that the right-hand side of (72) is $\mathrm{O}\left(t^{-m / 2}\right)$. Then, by Lemma 3, (31), (71) we have that the expression in (70) equals

$$
\begin{aligned}
& \int_{0}^{t} \mathrm{~d} s\left\{\int_{0}^{t} \mathrm{~d} \tau \frac{\mathrm{d}}{\mathrm{d} \tau}\left(\mathbb{P}_{x}\left[T_{K}<\tau\right]-\mathbb{P}_{x}\left[T_{K}<t\right]\right) \mathbb{P}_{x}\left[t-\tau<L_{K}<\infty\right]\right. \\
& \left.\quad-\int_{0}^{s} \mathrm{~d} \tau \frac{\mathrm{d}}{\mathrm{d} \tau}\left(\mathbb{P}_{x}\left[T_{K}<\tau\right]-\mathbb{P}_{x}\left[T_{K}<s\right]\right) \mathbb{P}_{x}\left[s-\tau<L_{K}<\infty\right]\right\} \int \mu_{K}(\mathrm{~d} y) p(x, y ; t-s)+\mathrm{O}\left(t^{-m / 2}\right) \\
& =\int_{0}^{t} \mathrm{~d} s\left\{\int_{s}^{t} \mathrm{~d} \tau \frac{\mathrm{d}}{\mathrm{d} \tau}\left(\mathbb{P}_{x}\left[T_{K}<\tau\right]-\mathbb{P}_{x}\left[T_{K}<t\right]\right) \mathbb{P}_{x}\left[t-\tau<L_{K}<\infty\right]\right.
\end{aligned}
$$




$$
\begin{aligned}
& \left.-\int_{0}^{s} \mathrm{~d} \tau \frac{\mathrm{d}}{\mathrm{d} \tau}\left(\mathbb{P}_{x}\left[T_{K}<\tau\right]-\mathbb{P}_{x}\left[T_{K}<s\right]\right) \mathbb{P}_{x}\left[s-\tau<L_{K}<t-\tau\right]\right\} \int \mu_{K}(\mathrm{~d} y) p(x, y ; t-s)+\mathrm{O}\left(t^{-m / 2}\right) \\
= & -\mathbb{P}_{x}\left[T_{K}<\infty\right] \int_{0}^{t} \mathrm{~d} s \mathbb{P}_{x}\left[s<L_{K}<t\right] \int \mu_{K}(\mathrm{~d} y) p(x, y ; t-s)+\sum_{i=1}^{4} A_{i}+\mathrm{O}\left(t^{-m / 2}\right),
\end{aligned}
$$

after two integrations by parts. Proposition 5 follows by (69) and (79).

Below we obtain the asymptotic behaviour of the first term in the right-hand side of (63).

Lemma 6. Let $K$ be a compact and non-polar set in $\mathbb{R}^{4}$. Then for $t \rightarrow \infty$

$$
\int_{0}^{t} \mathrm{~d} s \mathbb{P}_{x}\left[s<L_{K}<t\right] \int \mu_{K}(\mathrm{~d} y) p(x, y ; t-s)=2(4 \pi)^{-4} C(K)^{2} \frac{\log t}{t^{2}}+\mathrm{O}\left(t^{-2}\right) .
$$

\section{Proof. By (35)}

$$
\begin{aligned}
\int_{0}^{T} \mathrm{~d} s \mathbb{P}_{x}\left[s<L_{K}<t\right] \int \mu_{K}(\mathrm{~d} y) p(x, y ; t-s) & \leqslant \int_{0}^{T} \mathrm{~d} s \int \mu_{K}(\mathrm{~d} y) p(x, y ; t-s) \\
& \leqslant C(K) T(t-T)^{-2} .
\end{aligned}
$$

By (33)

$$
\begin{aligned}
\int_{t-T}^{t} \mathrm{~d} s \mathbb{P}_{x}\left[s<L_{K}<t\right] \int \mu_{K}(\mathrm{~d} y) p(x, y ; t-s) & \leqslant P_{x}\left[t-T<L_{K}<t\right] \int_{t-T}^{t} \mathrm{~d} s \int \mu_{K}(\mathrm{~d} y) p(x, y ; t-s) \\
& \leqslant \mathbb{P}_{x}\left[t-T<L_{K}<t\right] \leqslant C(K) T /(t(t-T)) .
\end{aligned}
$$

Furthermore by (35) and (19)

$$
\begin{aligned}
\int_{T}^{t-T} \mathrm{~d} s \mathbb{P}_{x}\left[s<L_{K}<t\right] \int \mu_{K}(\mathrm{~d} y) p(x, y ; t-s) & \leqslant(4 \pi)^{-4} C(K)^{2} \int_{T}^{t-T} \mathrm{~d} s\left(s^{-1}-t^{-1}\right)(t-s)^{-2} \\
& =2(4 \pi)^{-4} C(K)^{2} \frac{\log t}{t^{2}}+\mathrm{O}\left(t^{-2}\right),
\end{aligned}
$$

which proves the upper bound in (80). To prove the lower bound in (80) we have by (35) and (19)

$$
\begin{aligned}
& \int_{0}^{t} \mathrm{~d} s \mathbb{P}_{x}\left[s<L_{K}<t\right] \int \mu_{K}(\mathrm{~d} y) p(x, y ; t-s) \\
& \quad \geqslant \int_{T}^{t-T} \mathrm{~d} s \int_{s}^{t} \mathrm{~d} \tau(4 \pi \tau)^{-2} \int \mu_{K}(\mathrm{~d} z)\left(1-\frac{|x-z|^{2}}{4 \tau}\right) \int \mu_{K}(\mathrm{~d} y) p(x, y ; t-s) .
\end{aligned}
$$

Since

$$
\begin{gathered}
\int_{T}^{t-T} \mathrm{~d} s \int_{s}^{t} \mathrm{~d} \tau(4 \pi \tau)^{-2} \int \mu_{K}(\mathrm{~d} z) \frac{|x-z|^{2}}{4 \tau} \int \mu_{K}(\mathrm{~d} y) p(x, y ; t-s) \\
\leqslant C(K)^{2}(|x|+R)^{2} \int_{T}^{t-T} \mathrm{~d} s \int_{s}^{\infty} \mathrm{d} \tau \tau^{-3}(t-s)^{-2}=\mathrm{O}\left(t^{-2}\right)
\end{gathered}
$$


we have that the left-hand side of (84) is bounded below by

$$
\begin{aligned}
& \int_{T}^{t-T} \mathrm{~d} s \int_{s}^{t} \mathrm{~d} \tau(4 \pi \tau)^{-2} C(K) \int \mu_{K}(\mathrm{~d} y) p(x, y ; t-s)+\mathrm{O}\left(t^{-2}\right) \\
& \geqslant(4 \pi)^{-4} C(K)^{2} \int_{T}^{t-T} \mathrm{~d} s(t-s)^{-2}\left(s^{-1}-t^{-1}\right)-C(K)^{2}(|x|+R)^{2} \int_{T}^{t-T} \mathrm{~d} s(t-s)^{-3}\left(s^{-1}-t^{-1}\right)+\mathrm{O}\left(t^{-2}\right) \\
& =2(4 \pi)^{-4} C(K)^{2} \frac{\log t}{t^{2}}+\mathrm{O}\left(t^{-2}\right) .
\end{aligned}
$$

The lower bound in (80) follows from the estimates in (84)-(86).

We conclude this section with the proof of Proposition 2 for $m=4$. By (29) we have that the second term in the right-hand side of (48) is $\mathrm{O}\left(t^{-2}\right)$. Below we will show that $A_{i}=\mathrm{O}\left(t^{-2}\right)$ for $i=1, \ldots, 4$ and $t \rightarrow \infty$. This implies Theorem 1 for $m=4$ by Propositions 4, 5 and Lemma 6.

The contribution from $s \in[0, T]$ to $A_{1}$ in (64) is bounded by $C(K) T(t-T)^{-2}$. Similarly by (33) with $E=$ $[t-T, t]$ and (34) the contribution from $s \in[t-T, t]$ is bounded by

$$
\mathbb{P}_{x}\left[t-T<T_{K}<t\right] \int_{t-T}^{t} \mathrm{~d} s \int \mu_{K}(\mathrm{~d} y) p(x, y ; t-s) \leqslant C C(K) T /(t(t-T)) .
$$

The contribution from $s \in[T, t / 2]$ is bounded, using (29), by

$$
\int_{T}^{t / 2} \mathrm{~d} s C(K)^{3} s^{-1}(t-s)^{-3}=\mathrm{O}\left(\frac{\log t}{t^{3}}\right)
$$

and the contribution from $s \in[t / 2, t-T]$ is bounded, using (34) and (29), by

$$
\int_{t / 2}^{t-T} \mathrm{~d} s C C(K)^{2} s^{-2}(t-s)^{-2}=\mathrm{O}\left(t^{-2}\right) \text {. }
$$

This proves that $A_{1}=\mathrm{O}\left(t^{-2}\right)$.

The contribution from $s \in[0, T]$ to $A_{2}$ is bounded by $C(K) T(t-T)^{-2}$ and the contribution from $s \in[t-T, t]$ to $A_{2}$ is bounded, using (29), by $C(K)^{2}(t-T)^{-2}$.

Finally, the contribution from $s \in[T, t-T]$ is bounded, using (29), (30), by

$$
\int_{T}^{t-T} \mathrm{~d} s C(K)^{3} s^{-1}\left(s^{-1}-t^{-1}\right)(t-s)^{-2}=\mathrm{O}\left(t^{-2}\right) .
$$

This proves that $A_{2}=\mathrm{O}\left(t^{-2}\right)$.

The contribution from $s \in[0, t / 2]$ to $A_{3}$ is bounded, using Lemma 3 and (59), by

$$
t^{-2} \int_{0}^{t / 2} \mathrm{~d} s\left\{\int_{s}^{t / 2} \mathrm{~d} \tau \frac{C(K)^{3}}{\tau(t-\tau)^{2}}+\int_{t / 2}^{t-T} \mathrm{~d} \tau \frac{C C(K)^{2}}{\tau^{2}(t-\tau)}+\int_{t-T}^{t} \mathrm{~d} \tau \frac{C C(K) T}{(t-T)^{2}} \int \mu_{K}(\mathrm{~d} z) p(x, z ; t-\tau)\right\}=\mathrm{O}\left(\frac{\log t}{t^{3}}\right) .
$$

The contribution from $s \in[t / 2, t]$ to $A_{3}$ is bounded, using (34), by

$$
\int_{t / 2}^{t-T} \mathrm{~d} s \int_{s}^{t} \mathrm{~d} \tau \frac{C(t-\tau)}{\tau^{2}} \int \mu_{K}(\mathrm{~d} z) p(x, z ; t-\tau) \int \mu_{K}(\mathrm{~d} y) p(x, y ; t-s)
$$




$$
\begin{aligned}
& +\frac{C T}{(t-T)^{2}} \int_{t-T}^{t} \mathrm{~d} s \int_{t-T}^{t} \mathrm{~d} \tau \int \mu_{K}(\mathrm{~d} z) p(x, z ; t-\tau) \int \mu_{K}(\mathrm{~d} y) p(x, y ; t-s) \\
\leqslant & \frac{4 C}{t^{2}}\left(\int_{-\infty}^{t} \mathrm{~d} \tau(t-\tau)^{1 / 2} \int \mu_{K}(\mathrm{~d} z) p(x, z ; t-\tau)\right)^{2}+\frac{C T}{(t-T)^{2}} \\
= & \mathrm{O}\left(t^{-2}\right),
\end{aligned}
$$

where we have used that for $m=4$

$$
\begin{aligned}
& \int_{0}^{\infty} \mathrm{d} \tau \tau^{1 / 2} \int \mu_{K}(\mathrm{~d} y) p(x, y ; \tau)=\frac{1}{8 \pi^{3 / 2}} \int \mu_{K}(\mathrm{~d} y)|x-y|^{-1} \\
& \quad \leqslant \frac{1}{8 \pi^{3 / 2}}\left(\int \mu_{K}(\mathrm{~d} y)|x-y|^{-2}\right)^{1 / 2}\left(\int \mu_{K}(\mathrm{~d} y)\right)^{1 / 2} \leqslant C(K)^{1 / 2} .
\end{aligned}
$$

This proves that $A_{3}=\mathrm{O}\left(t^{-2}\right)$.

The contribution from $s \in[0,2 T]$ to $A_{4}$ is bounded by

$$
\frac{2 C(K) T}{(t-2 T)^{2}}\left(\int_{-\infty}^{s} \mathrm{~d} \tau \int \mu_{K}(\mathrm{~d} z) p(x, z ; s-\tau)+\int_{-\infty}^{t} \mathrm{~d} \tau \int \mu_{K}(\mathrm{~d} y) p(x, y ; t-\tau)\right)=\mathrm{O}\left(t^{-2}\right) .
$$

The contribution from $s \in[2 T, t / 2]$ to $A_{4}$ is bounded by

$$
\begin{aligned}
& C(K) t^{-2} \int_{2 T}^{t / 2} \mathrm{~d} s\left\{\int_{0}^{T} \mathrm{~d} \tau \frac{2 C(K)}{(s-\tau)^{2}}+\int_{T}^{s / 2} \mathrm{~d} \tau \frac{2 C(K)^{2}}{\tau(s-\tau)^{2}}+\int_{s / 2}^{s-T} \mathrm{~d} \tau \frac{2 C C(K)}{\tau^{2}(s-\tau)}+\int_{s-T}^{s} \mathrm{~d} \tau \frac{C T}{(s-T)^{2}}\right. \\
& \left.\quad \times \int \mu_{K}(\mathrm{~d} z)(p(x, z ; s-\tau)+p(x, z ; t-\tau))\right\}=\mathrm{O}\left(t^{-2}\right),
\end{aligned}
$$

where we have used that $P_{x}\left[\tau<T_{K}<s\right]$ is bounded on the intervals $[0, T],[T, s / 2],[s / 2, s-T]$ and $[s-T, s]$ by 1 , $C(K) / \tau, C(s-\tau) / \tau^{2}$ and $C T /(s-T)^{2}$ respectively.

To bound the contribution from $s \in[t / 2, t]$ to $A_{4}$ we use that uniformly in $x, z, s, \tau$ and $t$

$$
|p(x, z ; s-\tau)-p(x, z ; t-\tau)| \leqslant(s-\tau)^{-2} \wedge(t-s)(s-\tau)^{-3} \wedge(t-s)^{1 / 2}(s-\tau)^{-5 / 2} .
$$

First of all the contribution from the rectangle $\{(s, \tau): t / 2<s<t, 0<\tau<T\}$ to $A_{4}$ is bounded by

$$
\int_{t / 2}^{t} \mathrm{~d} s \int_{0}^{T} \mathrm{~d} \tau \frac{2 C(K)}{(s-\tau)^{2}} \int \mu_{K}(\mathrm{~d} y) p(x, y ; t-s) \leqslant \frac{2 C(K) T}{(t / 2-T)^{2}} \int_{t / 2}^{t} \mathrm{~d} s \int \mu_{K}(\mathrm{~d} y) p(x, y ; t-s)=\mathrm{O}\left(t^{-2}\right) .
$$

Secondly, by Lemma 3 and (96), (93)

$$
\begin{aligned}
& \int_{t / 2}^{t} \mathrm{~d} s \int_{T}^{s / 2} \mathrm{~d} \tau \mathbb{P}_{x}\left[\tau<T_{K}<s\right] \int \mu_{K}(\mathrm{~d} z)|p(x, z ; t-\tau)-p(x, z ; s-\tau)| \int \mu_{K}(\mathrm{~d} y) p(x, y ; t-s) \\
& \quad \leqslant \int_{t / 2}^{t} \mathrm{~d} s \int_{T}^{s / 2} \mathrm{~d} \tau \frac{C(K)^{2}(t-s)^{1 / 2}}{\tau(s-\tau)^{5 / 2}} \int \mu_{K}(\mathrm{~d} y) p(x, y ; t-s) \\
& \quad \leqslant C(K)^{2}\left(\frac{t}{4}\right)^{-5 / 2} \int_{t / 2}^{t} \mathrm{~d} s(t-s)^{1 / 2} \log \left(\frac{s}{2 T}\right) \int \mu_{K}(\mathrm{~d} y) p(x, y ; t-s)
\end{aligned}
$$




$$
\leqslant C(K)^{5 / 2}\left(\frac{t}{4}\right)^{-5 / 2} \log \left(\frac{t}{2 T}\right) .
$$

Thirdly, by Lemma 3 and (96), (93)

$$
\begin{aligned}
& \int_{t / 2}^{t} \mathrm{~d} s \int_{s / 2}^{s-T} \mathrm{~d} \tau \mathbb{P}_{x}\left[\tau<T_{K}<s\right] \int \mu_{K}(\mathrm{~d} z)|p(x, z ; t-\tau)-p(x, z ; s-\tau)| \int \mu_{K}(\mathrm{~d} y) p(x, y ; t-s) \\
& \leqslant \int_{t / 2}^{t} \mathrm{~d} s \int_{s / 2}^{s-T} \mathrm{~d} \tau \frac{C C(K)(t-s)^{1 / 2}}{\tau^{2}(s-\tau)^{3 / 2}} \int \mu_{K}(\mathrm{~d} y) p(x, y ; t-s) \\
& \leqslant 16 C C(K) t^{-2} \int_{0}^{t} \mathrm{~d} s(t-s)^{1 / 2} \int \mu_{K}(\mathrm{~d} y) p(x, y ; t-s) \\
& \quad \times \int_{-\infty}^{s-T} \mathrm{~d} \tau(s-\tau)^{-3 / 2} \leqslant 32 C C(K)^{3 / 2} T^{-1 / 2} t^{-2} .
\end{aligned}
$$

Finally, by Lemma 3,

$$
\begin{aligned}
& \int_{t / 2}^{t} \mathrm{~d} s \int_{s-T}^{s} \mathrm{~d} \tau \mathbb{P}_{x}\left[\tau<T_{K}<s\right] \int \mu_{K}(\mathrm{~d} z)|p(x, z ; t-\tau)-p(x, z ; s-\tau)| \int \mu_{K}(\mathrm{~d} y) p(x, y ; t-s) \\
& \quad \leqslant \int_{t / 2}^{t} \mathrm{~d} s \frac{C T}{(s-T)^{2}} \int_{s-T}^{s} \mathrm{~d} \tau \int \mu_{K}(\mathrm{~d} z)(p(x, z ; t-\tau)+p(x, z ; s-\tau)) \int \mu_{K}(\mathrm{~d} y) p(x, y ; t-s) \\
& \quad \leqslant \frac{8 C T}{(t-2 T)^{2}} .
\end{aligned}
$$

This completes the proof of $A_{4}=\mathrm{O}\left(t^{-2}\right)$ and hence of Proposition 2 for $m=4$.

\section{Proof of Proposition 2 for $m=3$}

Throughout this section we assume that $m=3$. By Propositions 4 and 5

$$
\begin{aligned}
& \mathbb{P}_{x}\left[t<T_{K}<\infty\right]=\left(1-\mathbb{P}_{x}\left[t<L_{K}<\infty\right]\right)^{-1} \mathbb{P}_{x}\left[T_{K}=\infty\right] \mathbb{P}_{x}\left[t<L_{K}<\infty\right] \\
& -\left(1-\mathbb{P}_{x}\left[t<L_{K}<\infty\right]\right)^{-1} \mathbb{P}_{x}\left[T_{K}=\infty\right] \int_{0}^{t} \mathrm{~d} s \mathbb{P}_{x}\left[s<L_{K}<t\right] \\
& \quad \times \int \mu_{K}(\mathrm{~d} y) p(x, y ; t-s)-\left(1-\mathbb{P}_{x}\left[t<L_{K}<\infty\right]\right)^{-1} \sum_{i=1}^{4} A_{i}+\mathrm{O}\left(t^{-3 / 2}\right) .
\end{aligned}
$$

By (20)

$$
\begin{aligned}
(1 & \left.-\mathbb{P}_{x}\left[t<L_{K}<\infty\right]\right)^{-1} \mathbb{P}_{x}\left[T_{K}=\infty\right] \mathbb{P}_{x}\left[t<L_{K}<\infty\right] \\
& =\mathbb{P}_{x}\left[T_{K}=\infty\right] \mathbb{P}_{x}\left[t<L_{K}<\infty\right]+\left(16 \pi^{3}\right)^{-1} C(K)^{2} \mathbb{P}_{x}\left[T_{K}=\infty\right] t^{-1}+\mathrm{O}\left(t^{-3 / 2}\right)
\end{aligned}
$$

Lemma 7. Let $K$ be a compact and non-polar set in $\mathbb{R}^{3}$. Then for $t \rightarrow \infty$

$$
\int_{0}^{t} \mathrm{~d} s \mathbb{P}_{x}\left[s<L_{K}<t\right] \int \mu_{K}(\mathrm{~d} y) p(x, y ; t-s)=\left(16 \pi^{3}\right)^{-1} C(K)^{2} t^{-1}+\mathrm{O}\left(t^{-3 / 2}\right) .
$$


Proof. By (19)

$$
\int \mu_{K}(\mathrm{~d} y) p(x, y ; t-s) \leqslant(4 \pi)^{-3 / 2} C(K)(t-s)^{-3 / 2},
$$

so that by (104) and (30)

$$
\begin{aligned}
\int_{0}^{t} \mathrm{~d} s \mathbb{P}_{x}\left[s<L_{K}<t\right] \int \mu_{K}(\mathrm{~d} y) p(x, y ; t-s) & \leqslant\left(32 \pi^{3}\right)^{-1} C(K)^{2} t^{-1} \int_{0}^{1} \mathrm{~d} s s^{-1 / 2}\left(1+s^{1 / 2}\right)^{-1}(1-s)^{-1 / 2} \\
& =\left(16 \pi^{3}\right)^{-1} C(K)^{2} t^{-1},
\end{aligned}
$$

where the integral with respect to $s \in[0,1]$ is evaluated by the change of variable $s=(\sin \theta)^{2}$. To prove the lower bound in Lemma 7 we have

$$
\int \mu_{K}(\mathrm{~d} y) p(x, y ; t-s) \geqslant(4 \pi)^{-3 / 2} C(K)(t-s)^{-3 / 2}-(4 \pi)^{-3 / 2} C(K)(t-s)^{-3 / 2}\left(1-\mathrm{e}^{-\frac{(|x|+R)^{2}}{4(t-s)}}\right) .
$$

Since

$$
\begin{aligned}
\int_{0}^{t} \mathrm{~d} s \mathbb{P}_{x}\left[s<L_{K}<t\right](t-s)^{-3 / 2}\left(1-\mathrm{e}^{-\frac{(|x|+R)^{2}}{4(t-s)}}\right) & \leqslant C(K) \int_{0}^{t} \mathrm{~d} s\left(s^{-1 / 2}-t^{-1 / 2}\right)(t-s)^{-3 / 2}\left(1-\mathrm{e}^{-\frac{(|x|+R)^{2}}{4(t-s)}}\right) \\
& =2 C(K) t^{-1} \int_{0}^{\pi / 2} \frac{\mathrm{d} \theta}{1+\sin \theta}\left(1-\mathrm{e}^{-\frac{(|x|+R)^{2}}{4 t(\cos \theta)^{2}}}\right) \\
& \leqslant 2 C(K) t^{-1} \int_{0}^{\pi / 2} \mathrm{~d} \theta\left(1-\mathrm{e}^{-\frac{(|x|+R)^{2}}{\theta^{2} t}}\right)=\mathrm{O}\left(t^{-3 / 2}\right),
\end{aligned}
$$

we have that the left-hand side of (103) is bounded from below by

$$
(4 \pi)^{-3 / 2} C(K) \int_{0}^{t} \mathrm{~d} s \mathbb{P}_{x}\left[s<L_{K}<t\right](t-s)^{-3 / 2}+\mathrm{O}\left(t^{-3 / 2}\right) .
$$

Since

$$
\begin{aligned}
\mathbb{P}_{x}\left[s<L_{K}<t\right] & \geqslant \int \mu_{K}(\mathrm{~d} y) \int_{s}^{t} \mathrm{~d} \tau(4 \pi \tau)^{-3 / 2} \mathrm{e}^{-\frac{(|x|+R)^{2}}{4 s}} \\
& =\left(4 \pi^{3 / 2}\right)^{-1} C(K)\left(s^{-1 / 2}-t^{-1 / 2}\right)\left(1-\left(1-\mathrm{e}^{-\frac{(|x|+R)^{2}}{4 s}}\right)\right),
\end{aligned}
$$

we have by estimates similar to (107) that (108) is bounded from below by

$$
\begin{aligned}
& \left(16 \pi^{3}\right)^{-1} C(K)^{2} t^{-1}-C(K)^{2} t^{-1} \int_{0}^{1} \mathrm{~d} s\left(s^{-1 / 2}-1\right)(1-s)^{-3 / 2}\left(1-\mathrm{e}^{-\frac{(|x|+R)^{2}}{4 s t}}\right)+\mathrm{O}\left(t^{-3 / 2}\right) \\
& =\left(16 \pi^{3}\right)^{-1} C(K)^{2} t^{-1}+\mathrm{O}\left(t^{-3 / 2}\right) .
\end{aligned}
$$

This completes, by (106)-(110), the proof of the lower bound in Lemma 7.

By Lemma 7 we obtain that the term of order $t^{-1}$ in (102) cancels with the second term in the right-hand side of (101). So the proof of Proposition 2 for $m=3$ is complete if we can show that

$$
\sum_{i=1}^{4} A_{i}=\mathrm{O}\left(t^{-3 / 2}\right), \quad t \rightarrow \infty \text {. }
$$


However, it turns out that each of the $A_{i}$ is (for $m=3$ ) of order $(\log t) / t^{3 / 2}$. So in order to obtain (111) we will show that the sum of the coefficients of $\log t / t^{3 / 2}$ of the $A_{i}$ 's cancel with remainder $\mathrm{O}\left(t^{-3 / 2}\right)$. In Proposition 8 we state that $\mathbb{P}_{x}\left[s<T_{K}<t\right]$ in (64) can be replaced by $\mathbb{P}_{x}\left[s<L_{K}<t\right] \mathbb{P}_{x}\left[T_{K}=\infty\right]$ at a cost of $\mathrm{O}\left(t^{-3 / 2}\right)$ with similar replacements in (65)-(67) respectively. In Lemma 9 we obtain, using Proposition 8 , the desired asymptotic behaviour of each of the $A_{i}$. This in turn implies (111) and thereby completing the proof of (111) and of Theorem 1.

Proposition 8. Let $K$ be a compact, non-polar set in $\mathbb{R}^{3}$, and let $A_{i} i=1, \ldots, 4$ be given by (64)-(67) respectively. Then for $t \rightarrow \infty$

$$
\begin{aligned}
A_{1}= & \mathbb{P}_{x}\left[T_{K}=\infty\right] \int_{0}^{t} \mathrm{~d} s \mathbb{P}_{x}\left[s<L_{K}<t\right] \mathbb{P}_{x}\left[t-s<L_{K}<\infty\right] \int \mu_{K}(\mathrm{~d} y) p(x, y ; t-s)+\mathrm{O}\left(t^{-3 / 2}\right), \\
A_{2}= & \mathbb{P}_{x}\left[T_{K}=\infty\right] \int_{0}^{t} \mathrm{~d} s \mathbb{P}_{x}\left[s<L_{K}<\infty\right] \mathbb{P}_{x}\left[s<L_{K}<t\right] \int \mu_{K}(\mathrm{~d} y) p(x, y ; t-s)+\mathrm{O}\left(t^{-3 / 2}\right), \\
A_{3}= & \mathbb{P}_{x}\left[T_{K}=\infty\right] \int_{0}^{t} \mathrm{~d} s \int_{s}^{t} \mathrm{~d} \tau \mathbb{P}_{x}\left[\tau<L_{K}<t\right] \int \mu_{K}(\mathrm{~d} z) p(x, z ; t-\tau) \\
& \times \int \mu_{K}(\mathrm{~d} y) p(x, y ; t-s)+\mathrm{O}\left(t^{-3 / 2}\right), \\
A_{4}= & \mathbb{P}_{x}\left[T_{K}=\infty\right] \int_{0}^{t} \mathrm{~d} s \int_{0}^{s} \mathrm{~d} \tau \mathbb{P}_{x}\left[\tau<L_{K}<s\right] \int \mu_{K}(\mathrm{~d} z) \\
& \times(p(x, z ; t-\tau)-p(x, z ; s-\tau)) \int \mu_{K}(\mathrm{~d} y) p(x, y ; t-s)+\mathrm{O}\left(t^{-3 / 2}\right) .
\end{aligned}
$$

It is convenient to denote the first term in the right-hand sides of (112)-115) respectively by $B_{1}, \ldots, B_{4}$.

Lemma 9. Let $K$ be a compact and non-polar set in $\mathbb{R}^{3}$. Then for $t \rightarrow \infty$

$$
\begin{aligned}
& B_{1}=2(4 \pi)^{-9 / 2} C(K)^{3} \mathbb{P}_{x}\left[T_{K}=\infty\right] \frac{\log t}{t^{3 / 2}}+\mathrm{O}\left(t^{-3 / 2}\right), \\
& B_{2}=4(4 \pi)^{-9 / 2} C(K)^{3} \mathbb{P}_{x}\left[T_{K}=\infty\right] \frac{\log t}{t^{3 / 2}}+\mathrm{O}\left(t^{-3 / 2}\right), \\
& B_{3}=2(4 \pi)^{-9 / 2} C(K)^{3} \mathbb{P}_{x}\left[T_{K}=\infty\right] \frac{\log t}{t^{3 / 2}}+\mathrm{O}\left(t^{-3 / 2}\right), \\
& B_{4}=-8(4 \pi)^{-9 / 2} C(K)^{3} \mathbb{P}_{x}\left[T_{K}=\infty\right] \frac{\log t}{t^{3 / 2}}+\mathrm{O}\left(t^{-3 / 2}\right) .
\end{aligned}
$$

Proof. By (29), (30) and (104)

$$
B_{1} \leqslant 4(4 \pi)^{-9 / 2} C(K)^{2} \mathbb{P}_{x}\left[T_{K}=\infty\right] \int_{0}^{t} \mathrm{~d} s\left(s^{-1 / 2}-t^{-1 / 2}\right)(t-s)^{-2} \int \mu_{K}(\mathrm{~d} y) \mathrm{e}^{-\frac{|x-y|^{2}}{4(t-s)}} .
$$

On the other hand, by (35)

$$
\mathbb{P}_{x}\left[t-s<L_{K}<\infty\right] \geqslant 2(4 \pi)^{-3 / 2} C(K)(t-s)^{-1 / 2}\left(1+\mathrm{e}^{-\frac{(|x|+R)^{2}}{4(t-s)}}-1\right) .
$$

Hence by (109) and (121) 


$$
\begin{aligned}
B_{1} \geqslant & 4(4 \pi)^{-9 / 2} C(K)^{2} \mathbb{P}_{x}\left[T_{K}=\infty\right] \int_{0}^{t} \mathrm{~d} s\left(s^{-1 / 2}-t^{-1 / 2}\right)(t-s)^{-2} \\
& \times \int \mu_{K}(\mathrm{~d} y) \mathrm{e}^{-\frac{|x-y|^{2}}{4(t-s)}}\left(1-\left(1-\mathrm{e}^{-\frac{(|x|+R)^{2}}{4(t-s)}}\right)-\left(1-\mathrm{e}^{-\frac{(|x|+R)^{2}}{4 s}}\right)\right) .
\end{aligned}
$$

Below we will compute the leading asymptotic behaviour of the right-hand side of (120). Substitution of $s=t(\cos \theta)^{2}$ in (120) yields that the integral equals

$$
2 t^{-3 / 2} \int \mu_{K}(\mathrm{~d} y) \int_{0}^{\pi / 2} \mathrm{~d} \theta(\sin \theta)^{-1}(1+\cos \theta)^{-1} \mathrm{e}^{-\frac{|x-y|^{2}}{4 t(\sin \theta)^{2}}} .
$$

Since

$$
(\sin \theta)^{-1}(1+\cos \theta)^{-1} \leqslant(2 \theta)^{-1}+4, \quad 0<\theta<\pi / 2,
$$

we have that the right-hand side of (123) is bounded from above by

$$
\begin{aligned}
& t^{-3 / 2} \int \mu_{K}(\mathrm{~d} y) \int_{0}^{\pi / 2} \mathrm{~d} \theta \theta^{-1} \mathrm{e}^{-\frac{|x-y|^{2}}{4 t \theta^{2}}}+\mathrm{O}\left(t^{-3 / 2}\right)=\frac{1}{2} t^{-3 / 2} \int \mu_{K}(\mathrm{~d} y) \int_{\frac{|x-y|^{2}}{\pi^{2} t}}^{\infty} \mathrm{d} u u^{-1} \mathrm{e}^{-u}+\mathrm{O}\left(t^{-3 / 2}\right) \\
& =\frac{1}{2} t^{-3 / 2} \int \mu_{K}(\mathrm{~d} y) \log \left(\frac{|x-y|^{2}}{\pi^{2} t}\right)+\mathrm{O}\left(t^{-3 / 2}\right) .
\end{aligned}
$$

This gives, together with (120), the desired upper bound for the asymptotic behaviour of the right-hand side of (120). The lower bound for the right-hand side of (120) follows similarly, using $(\sin \theta)^{-1}(1+\cos \theta)^{-1} \geqslant$ $(2 \theta)^{-1}, 0<\theta<\pi / 2$. Furthermore returning to (122) we have that

$$
\begin{aligned}
& \int_{0}^{t} \mathrm{~d} s\left(s^{-1 / 2}-t^{-1 / 2}\right)(t-s)^{-2} \int \mu_{K}(\mathrm{~d} y) \mathrm{e}^{-\frac{|x-y|^{2}}{4(t-s)}}\left(1-\mathrm{e}^{-\frac{(|x|+R)^{2}}{4(t-s)}}\right) \\
& \leqslant 2 t^{-3 / 2} \int_{0}^{\pi / 2} \mathrm{~d} \theta(\sin \theta)^{-1} \int \mu_{K}(\mathrm{~d} y) \mathrm{e}^{-\frac{|x-y|^{2}}{4 t(\sin \theta)^{2}}}\left(1-\mathrm{e}^{-\frac{(|x|+R)^{2}}{4 t(\sin \theta)^{2}}}\right) \\
& \leqslant 2 t^{-3 / 2} \int_{0}^{\pi / 2} \mathrm{~d} \theta(\sin \theta)^{-1} \int \mu_{K}(\mathrm{~d} y)\left(\frac{4 t(\sin \theta)^{2}}{|x-y|^{2}}\right)^{1 / 2}\left(1-\mathrm{e}^{-\frac{(|x|+R)^{2}}{4 t(\sin \theta)^{2}}}\right) \\
& \leqslant 16 \pi t^{-1}\left(\int \mu_{K}(\mathrm{~d} y) \frac{1}{4 \pi|x-y|}\right) \int_{0}^{\infty} \mathrm{d} \theta\left(1-\mathrm{e}^{-\frac{\pi^{2}(|x|+R)^{2}}{16 t \theta \theta^{2}}}\right)=\mathrm{O}\left(t^{-3 / 2}\right),
\end{aligned}
$$

and

$$
\begin{aligned}
& \int_{0}^{t} \mathrm{~d} s\left(s^{-1 / 2}-t^{-1 / 2}\right)(t-s)^{-2} \int \mu_{K}(\mathrm{~d} y) \mathrm{e}^{-\frac{|x-y|^{2}}{4(t-s)}}\left(1-\mathrm{e}^{-\frac{(|x|+R)^{2}}{4 s}}\right) \\
& \leqslant \int_{0}^{t} \mathrm{~d} s\left(s^{-1 / 2}-t^{-1 / 2}\right)(t-s)^{-2} \int \mu_{K}(\mathrm{~d} y)\left(\frac{4(t-s)}{|x-y|^{2}}\right)^{1 / 2}\left(1-\mathrm{e}^{-\frac{(|x|+R)^{2}}{4 s}}\right) \\
& \leqslant 8 \pi t^{-1} \int_{0}^{t} \mathrm{~d} s s^{-1 / 2}(t-s)^{-1 / 2}\left(1-\mathrm{e}^{-\frac{(|x|+R)^{2}}{4 s}}\right)
\end{aligned}
$$




$$
=16 \pi t^{-1} \int_{0}^{\pi / 2} \mathrm{~d} \theta\left(1-\mathrm{e}^{-\frac{(|x|+R)^{2}}{4 t(\sin \theta)^{2}}}\right)=\mathrm{O}\left(t^{-3 / 2}\right) .
$$

It follows by (126) and (127) that the two remainders in the right-hand side of (122) contribute each at most $\mathrm{O}\left(t^{-3 / 2}\right)$. This completes the proof of (116).

To prove (117) we note that by (29), (30) and (104)

$$
\begin{aligned}
B_{2} & \leqslant 2(4 \pi)^{-9 / 2} C(K)^{2} \mathbb{P}_{x}\left[T_{K}=+\infty\right] \int_{0}^{t} \mathrm{~d} s\left(s^{-1 / 2}-t^{-1 / 2}\right)(t-s)^{-3 / 2} \int_{K}(\mathrm{~d} y) \int_{s}^{\infty} \mathrm{d} \tau \tau^{-3 / 2} \mathrm{e}^{-\frac{|x-y|^{2}}{4 \tau}} \\
& =4(4 \pi)^{-9 / 2} C(K)^{2} \mathbb{P}_{x}\left[T_{K}=\infty\right] t^{-3 / 2} \int_{0}^{\pi / 2} \frac{\mathrm{d} \theta}{\sin \theta(1+\sin \theta)} \int \mu_{K}(\mathrm{~d} y) \int_{1}^{\infty} \mathrm{d} \tau \tau^{-3 / 2} \mathrm{e}^{-\frac{|x-y|^{2}}{4 \tau t(\sin \theta)^{2}}} .
\end{aligned}
$$

On the other hand

$$
\begin{aligned}
B_{2} \geqslant & 2(4 \pi)^{-9 / 2} C(K)^{2} \mathbb{P}_{x}\left[T_{K}=\infty\right] \int_{0}^{t} \mathrm{~d} s\left(s^{-1 / 2}-t^{-1 / 2}\right)(t-s)^{-3 / 2} \\
& \times \int \mu_{K}(\mathrm{~d} y) \int_{s}^{t} \mathrm{~d} \tau \tau^{-3 / 2} \mathrm{e}^{-\frac{|x-y|^{2}}{4 \tau}}\left(1-\left(1-\mathrm{e}^{-\frac{(|x|+R)^{2}}{4 s}}\right)-\left(1-\mathrm{e}^{-\frac{(|x|+R)^{2}}{4(t-s)}}\right)\right) .
\end{aligned}
$$

Below we will compute the leading asymptotic behaviour of the right-hand side of (128). Using the inequality $(\sin \theta)^{-1} \leqslant \theta^{-1}+4,0<\theta<\pi / 2$, we obtain for (128) the upper bound

$$
4(4 \pi)^{-9 / 2} C(K)^{2} \mathbb{P}_{x}\left[T_{K}=\infty\right] t^{-3 / 2} \int_{0}^{\pi / 2} \mathrm{~d} \theta \theta^{-1} \int_{1}^{\infty} \mathrm{d} \tau \tau^{-3 / 2} \int \mu_{K}(\mathrm{~d} y) \mathrm{e}^{-\frac{|x-y|^{2}}{4 \tau t \theta^{2}}}+\mathrm{O}\left(t^{-3 / 2}\right),
$$

and the upper bound follows by a calculation similar to (125). The lower bound for the right-hand side of (128) follows using $(\sin \theta)^{-1}(1+\sin \theta)^{-1} \geqslant \theta^{-1}-4,0<\theta<\pi / 2$. Furthermore returning to (129) we have a first error term

$$
\int_{0}^{t} \mathrm{~d} s\left(s^{-1 / 2}-t^{-1 / 2}\right)(t-s)^{-3 / 2}\left(1-\mathrm{e}^{-\frac{(|x|+R)^{2}}{4 s}}\right) \int \mu_{K}(\mathrm{~d} y) \int_{s}^{t} \mathrm{~d} \tau \tau^{-3 / 2} \mathrm{e}^{-\frac{|x-y|^{2}}{4 \tau}} .
$$

Since

$$
\begin{aligned}
\int \mu_{K}(\mathrm{~d} y) \int_{s}^{t} \mathrm{~d} \tau \tau^{-3 / 2} \mathrm{e}^{-\frac{|x-y|^{2}}{4 \tau}} & \leqslant \int \mu_{K}(\mathrm{~d} y) \int_{s}^{\infty} \mathrm{d} \tau \tau^{-3 / 2}\left(\frac{4 \tau}{|x-y|^{2}}\right)^{1 / 4} \\
& \leqslant 4 \sqrt{2} s^{-1 / 4} \int \mu_{K}(\mathrm{~d} y)|x-y|^{-1 / 2} \\
& \leqslant 4 \sqrt{2} s^{-1 / 4}\left(\int \mu_{K}(\mathrm{~d} y)|x-y|^{-1}\right)^{1 / 2} C(K)^{1 / 2} \\
& \leqslant 8 \sqrt{2 \pi} s^{-1 / 4} C(K)^{1 / 2},
\end{aligned}
$$

we have that (131) is bounded from above by

$$
8 \sqrt{2 \pi} C(K)^{1 / 2} t^{-1} \int_{0}^{t} \mathrm{~d} s s^{-3 / 4}(t-s)^{-1 / 2}\left(1-\mathrm{e}^{-\frac{(|x|+R)^{2}}{4 s}}\right)
$$




$$
\begin{aligned}
& \leqslant 16 \sqrt{2 \pi} C(K)^{1 / 2} t^{-5 / 4} \int_{0}^{\pi / 2} \mathrm{~d} \theta(\sin \theta)^{-1 / 2}\left(1-\mathrm{e}^{-\frac{(|x|+R)^{2}}{4 t(\sin \theta)^{2}}}\right) \\
& \leqslant 16 \pi C(K)^{1 / 2} t^{-5 / 4} \int_{0}^{\infty} \mathrm{d} \theta \theta^{-1 / 2}\left(1-\mathrm{e}^{-\frac{(|x|+R)^{2}}{4 t \theta^{2}}}\right)=\mathrm{O}\left(t^{-3 / 2}\right) .
\end{aligned}
$$

The second error term is bounded by

$$
\begin{aligned}
& \int_{0}^{t} \mathrm{~d} s\left(s^{-1 / 2}-t^{-1 / 2}\right)(t-s)^{-3 / 2}\left(1-\mathrm{e}^{-\frac{(|x|+R)^{2}}{4(t-s)}}\right) \int \mu_{K}(\mathrm{~d} y) \int_{s}^{t} \mathrm{~d} \tau \tau^{-3 / 2} \mathrm{e}^{-\frac{|x-y|^{2}}{4 \tau}} \\
& \leqslant 8 \sqrt{2 \pi} C(K)^{1 / 2} t^{-1} \int_{0}^{t} \mathrm{~d} s s^{-3 / 4}(t-s)^{-1 / 2}\left(1-\mathrm{e}^{-\frac{(|x|+R)^{2}}{4(t-s)}}\right) \\
& \leqslant 8 \sqrt{\pi} C(K)^{1 / 2}(|x|+R)^{1 / 2} t^{-1} \int_{0}^{t} \mathrm{~d} s s^{-3 / 4}(t-s)^{-3 / 4}=\mathrm{O}\left(t^{-3 / 2}\right)
\end{aligned}
$$

where we have used (132) and the inequality $1-\mathrm{e}^{-\theta} \leqslant \theta^{1 / 4}, \theta \geqslant 0$. It follows by (133) and (134) that the two remainders in the right-hand side of (129) contribute each at most $\mathrm{O}\left(t^{-3 / 2}\right)$. This completes the proof of (117).

To prove (118) we note that by (30) and (104)

$$
\begin{aligned}
B_{3} \leqslant & 2(4 \pi)^{-9 / 2} C(K)^{2} \mathbb{P}_{x}\left[T_{K}=\infty\right] \int_{0}^{t} \mathrm{~d} s \int \mu_{K}(\mathrm{~d} y) \mathrm{e}^{-\frac{|x-y|^{2}}{4(t-s)}}(t-s)^{-3 / 2} \\
& \times \int_{s}^{t} \mathrm{~d} \tau\left(\tau^{-1 / 2}-t^{-1 / 2}\right)(t-\tau)^{-3 / 2} .
\end{aligned}
$$

On the other hand

$$
\begin{aligned}
B_{3} \geqslant & 2(4 \pi)^{-9 / 2} C(K)^{2} \mathbb{P}_{x}\left[T_{K}=\infty\right] \int_{0}^{t} \mathrm{~d} s \int \mu_{K}(\mathrm{~d} y) \mathrm{e}^{-\frac{|x-y|^{2}}{4(t-s)}}(t-s)^{-3 / 2} \int_{s}^{t} \mathrm{~d} \tau\left(\tau^{-1 / 2}-t^{-1 / 2}\right)(t-\tau)^{-3 / 2} \\
& \times\left(1-\left(1-\mathrm{e}^{-\frac{(|x|+R)^{2}}{4 s}}\right)-\left(1-\mathrm{e}^{-\frac{(|x|+R)^{2}}{4(t-s)}}\right)\right) .
\end{aligned}
$$

Below we will compute the leading asymptotic behaviour of the right-hand side of (135). Firstly, since

$$
\int_{s}^{t} \mathrm{~d} \tau\left(\tau^{-1 / 2}-t^{-1 / 2}\right)(t-\tau)^{-3 / 2}=\int_{s}^{t} \mathrm{~d} \tau \tau^{-1 / 2} t^{-1 / 2}\left(\tau^{1 / 2}+t^{1 / 2}\right)^{-1}(t-\tau)^{-1 / 2} \geqslant \frac{(t-s)^{1 / 2}}{t^{3 / 2}}
$$

we have that the right-hand side of (135) is bounded from below by

$$
\begin{gathered}
2(4 \pi)^{-9 / 2} C(K)^{2} \mathbb{P}_{x}\left[T_{K}=\infty\right] t^{-3 / 2} \int_{0}^{t} \mathrm{~d} s \int \mu_{K}(\mathrm{~d} y)(t-s)^{-1} \mathrm{e}^{-\frac{|x-y|^{2}}{4(t-s)}} \\
\geqslant 2(4 \pi)^{-9 / 2} C(K)^{3} \mathbb{P}_{x}\left[T_{K}=\infty\right] \frac{\log t}{t^{3 / 2}}+\mathrm{O}\left(t^{-3 / 2}\right) .
\end{gathered}
$$

Secondly, since

$$
\int_{s}^{t} \mathrm{~d} \tau\left(\tau^{-1 / 2}-t^{-1 / 2}\right)(t-\tau)^{-3 / 2} \leqslant \frac{(t-s)^{1 / 2}}{t^{3 / 2}}+\frac{2(t-s)^{3 / 2}}{t^{2} s^{1 / 2}}
$$


we have that the right-hand side of (135) is bounded from above by

$$
\begin{aligned}
& 2(4 \pi)^{-9 / 2} C(K)^{2} \mathbb{P}_{x}\left[T_{K}=\infty\right] t^{-3 / 2} \int_{0}^{t} \mathrm{~d} s \int \mu_{K}(\mathrm{~d} y)(t-s)^{-1} \mathrm{e}^{-\frac{|x-y|^{2}}{4(t-s)}}+\mathrm{O}\left(t^{-3 / 2}\right) \\
& \leqslant 2(4 \pi)^{-9 / 2} C(K)^{3} \mathbb{P}_{x}\left[T_{K}=\infty\right] \frac{\log t}{t^{3 / 2}}+\mathrm{O}\left(t^{-3 / 2}\right) .
\end{aligned}
$$

In order to complete the proof of (118) we have to show that the two error terms in the right-hand side of (136) contribute at most $\mathrm{O}\left(t^{-3 / 2}\right)$. Since the right-hand side of (139) is bounded from above by $3(t-s)^{1 / 2} t^{-1} s^{-1 / 2}$ we have that the first of these error terms is bounded by

$$
\begin{aligned}
& C(K)^{2} t^{-1} \int_{0}^{t} \mathrm{~d} s \int \mu_{K}(\mathrm{~d} y)(t-s)^{-1} s^{-1 / 2} \mathrm{e}^{-\frac{|x-y|^{2}}{4(t-s)}}\left(1-\mathrm{e}^{-\frac{(|x|+R)^{2}}{4 s}}\right) \\
& \leqslant 8 \pi C(K)^{2} t^{-1} \int_{K}(\mathrm{~d} y)(4 \pi|x-y|)^{-1} \int_{0}^{t} \mathrm{~d} s(t-s)^{-1 / 2} s^{-1 / 2}\left(1-\mathrm{e}^{-\frac{(|x|+R)^{2}}{4 s}}\right) \\
& \leqslant 16 \pi C(K)^{2} t^{-1} \int_{0}^{\pi / 2} \mathrm{~d} \theta\left(1-\mathrm{e}^{-\frac{(|x|+R)^{2}}{4 t(\sin \theta)^{2}}}\right)=\mathrm{O}\left(t^{-3 / 2}\right) .
\end{aligned}
$$

The upper bound for the second of these error terms follows by a similar calculation. This completes the proof of (118).

To prove (119) we rewrite $B_{4}$ as follows.

$$
\begin{aligned}
B_{4}= & (4 \pi)^{-3 / 2} C(K) \mathbb{P}_{x}\left[T_{K}=\infty\right] \int_{0}^{t} \mathrm{~d} s \int \mu_{K}(\mathrm{~d} y) p(x, y ; t-s) \\
& \times \int_{0}^{s} \mathrm{~d} \tau\left((t-\tau)^{-3 / 2}-(s-\tau)^{-3 / 2}\right) \int_{\tau}^{s} \mathrm{~d} \rho \int \mu_{K}(\mathrm{~d} w) p(x, w ; \rho) \\
& +(4 \pi)^{-3 / 2} \mathbb{P}_{x}\left[T_{K}=\infty\right] \int_{0}^{t} \mathrm{~d} s \int \mu_{K}(\mathrm{~d} y) p(x, y ; t-s) \int \mu_{K}(\mathrm{~d} z) \\
& \times \int_{0}^{s} \mathrm{~d} \tau(t-\tau)^{-3 / 2}\left(\mathrm{e}^{-\frac{|x-z|^{2}}{4(t-\tau)}}-1\right) \int_{\tau}^{s} \mathrm{~d} \rho \int \mu_{K}(\mathrm{~d} w) p(x, w ; \rho) \\
& +(4 \pi)^{-3 / 2} \mathbb{P}_{x}\left[T_{K}=\infty\right] \int_{0}^{t} \mathrm{~d} s \int \mu_{K}(\mathrm{~d} y) p(x, y ; t-s) \int \mu_{K}(\mathrm{~d} z) \\
& \times \int_{0}^{s} \mathrm{~d} \tau(s-\tau)^{-3 / 2}\left(1-\mathrm{e}^{-\frac{|x-z|^{2}}{4(s-\tau)}}\right) \int_{\tau}^{s} \mathrm{~d} \rho \int \mu_{K}(\mathrm{~d} w) p(x, w ; \rho) .
\end{aligned}
$$

We first show that the third term in the right-hand side of (142) is bounded in absolute value by $\mathrm{O}\left(t^{-3 / 2}\right)$. Note that

$$
\begin{aligned}
\int_{\tau}^{s} \mathrm{~d} \rho \int \mu_{K}(\mathrm{~d} w) p(x, w ; \rho) & \leqslant 2(4 \pi)^{-3 / 2}\left(\tau^{-1 / 2}-s^{-1 / 2}\right) \int \mu_{K}(\mathrm{~d} w) \mathrm{e}^{-\frac{|x-w|^{2}}{4 s}} \\
& \leqslant(s-\tau) \tau^{-1 / 2} s^{-1} \int \mu_{K}(\mathrm{~d} w) \mathrm{e}^{-\frac{|x-w|^{2}}{4 s}}
\end{aligned}
$$


Hence the absolute value of this third term is bounded by

$$
\begin{aligned}
& C(K) \int_{0}^{t} \mathrm{~d} s \int \mu_{K}(\mathrm{~d} y) p(x, y ; t-s) \int_{0}^{s} \mathrm{~d} \tau\left(1-\mathrm{e}^{-\frac{(|x|+R)^{2}}{4(s-\tau)}}\right)(s-\tau)^{-1 / 2} \tau^{-1 / 2} s^{-1} \int \mu_{K}(\mathrm{~d} w) \mathrm{e}^{-\frac{|x-w|^{2}}{4 s}} \\
& \quad=2 C(K) \int_{0}^{t} \mathrm{~d} s \int \mu_{K}(\mathrm{~d} y) p(x, y ; t-s) s^{-1} \int_{0}^{\pi / 2} \mathrm{~d} \theta\left(1-\mathrm{e}^{-\frac{(|x|+R)^{2}}{4 s(\sin \theta)^{2}}}\right) \int \mu_{K}(\mathrm{~d} w) \mathrm{e}^{-\frac{|x-w|^{2}}{4 s}} \\
& \leqslant(4 \pi)^{2}(|x|+R) C(K) \int_{0}^{t} \mathrm{~d} s \int \mu_{K}(\mathrm{~d} y) p(x, y ; t-s) \int \mu_{K}(\mathrm{~d} w) p(x, w ; s)=\mathrm{O}\left(t^{-3 / 2}\right) .
\end{aligned}
$$

Since for $0<\tau<s<t$

$$
(t-\tau)^{-3 / 2}\left(1-\mathrm{e}^{-\frac{|x-z|^{2}}{4(t-\tau)}}\right) \leqslant(s-\tau)^{-3 / 2}\left(1-\mathrm{e}^{-\frac{(|x|+R)^{2}}{4(s-\tau)}}\right),
$$

we have that the second term in the right-hand side of (142) is also estimated by (144).

It remains to find the asymptotic behaviour of the first term in the right-hand side of (142). By the first inequality in (143) we have that this term is bounded from below by

$$
\begin{aligned}
& 2(4 \pi)^{-9 / 2} C(K) \mathbb{P}_{x}\left[T_{K}=\infty\right] \int_{0}^{t} \mathrm{~d} s \int \mu_{K}(\mathrm{~d} y)(t-s)^{-3 / 2} \mathrm{e}^{-\frac{|x-y|^{2}}{4(t-s)}} \\
& \quad \times \int_{0}^{s} \mathrm{~d} \tau\left((t-\tau)^{-3 / 2}-(s-\tau)^{-3 / 2}\right)\left(\tau^{-1 / 2}-s^{-1 / 2}\right) \int \mu_{K}(\mathrm{~d} w) \mathrm{e}^{-\frac{|x-w|^{2}}{4 s}} .
\end{aligned}
$$

A straightforward calculation gives that

$$
\begin{aligned}
& \int_{0}^{s} \mathrm{~d} \tau\left((s-\tau)^{-3 / 2}-(t-\tau)^{-3 / 2}\right)\left(\tau^{-1 / 2}-s^{-1 / 2}\right) \\
& \quad=2(t-s)^{3 / 2}\left(t^{1 / 2}+(t-s)^{1 / 2}\right)^{-1} s^{-1}\left[(t-s)^{-1}+\left(t+s+(s t)^{1 / 2}\right) t^{-1}(t-s)^{-1 / 2}\left(t^{1 / 2}+s^{1 / 2}\right)^{-1}\right] .
\end{aligned}
$$

Hence (146) equals

$$
\begin{aligned}
& -4(4 \pi)^{-9 / 2} C(K) \mathbb{P}_{x}\left[T_{K}=\infty\right] \int_{0}^{t} \mathrm{~d} s \int \mu_{K}(\mathrm{~d} y) \int \mu_{K}(\mathrm{~d} w) \mathrm{e}^{-\frac{|x-y|^{2}}{4(t-s)}-\frac{|x-w|^{2}}{4 s}}\left(t^{1 / 2}+(t-s)^{1 / 2}\right)^{-1} s^{-1} \\
& \times\left[(t-s)^{-1}+\left(t+s+(s t)^{1 / 2}\right) t^{-1}(t-s)^{-1 / 2}\left(t^{1 / 2}+s^{1 / 2}\right)^{-1}\right] .
\end{aligned}
$$

The first term in the square brackets of (148) gives the contribution

$$
-6(4 \pi)^{-9 / 2} C(K)^{3} \mathbb{P}_{x}\left[T_{K}=\infty\right] \frac{\log t}{t^{3 / 2}}+\mathrm{O}\left(t^{-3 / 2}\right)
$$

and the second term contributes

$$
-2(4 \pi)^{-9 / 2} C(K)^{3} \mathbb{P}_{x}\left[T_{K}=\infty\right] \frac{\log t}{t^{3 / 2}}+\mathrm{O}\left(t^{-3 / 2}\right)
$$

By (146)-(150) we conclude that the first term in the right-hand side of (142) is bounded from below by the expression in the right-hand side of (119). Since

$$
\int_{\tau}^{s} \mathrm{~d} \rho \int \mu_{K}(\mathrm{~d} w) p(x, w ; \rho) \geqslant 2(4 \pi)^{-3 / 2}\left(\tau^{-1 / 2}-s^{-1 / 2}\right) \int \mu_{K}(\mathrm{~d} w) \mathrm{e}^{-\frac{|x-w|^{2}}{4 \tau}}
$$


we have, by (143), that the resulting upper bound differs from the lower bound by at most

$$
\begin{aligned}
& \int_{0}^{t} \mathrm{~d} s \int_{K} \mu_{K}(\mathrm{~d} y) p(x, y ; t-s) \int_{0}^{s} \mathrm{~d} \tau\left((s-\tau)^{-3 / 2}-(t-\tau)^{-3 / 2}\right) \\
& \quad \times C(K)\left(\tau^{-1 / 2}-s^{-1 / 2}\right) \int \mu_{K}(\mathrm{~d} w)\left(\mathrm{e}^{-\frac{|x-w|^{2}}{4 s}}-\mathrm{e}^{-\frac{|x-w|^{2}}{4 \tau}}\right) \\
& \leqslant \int_{0}^{t} \mathrm{~d} s \int \mu_{K}(\mathrm{~d} y) p(x, y ; t-s) \int \mu_{K}(\mathrm{~d} w) s^{-1} \mathrm{e}^{-\frac{|x-w|^{2}}{4 s}} \\
& \quad \times C(K) \int_{0}^{s} \mathrm{~d} \tau \tau^{-1 / 2}(s-\tau)^{-1 / 2}\left(1-\mathrm{e}^{-|x-w|^{2}\left(\frac{1}{4 \tau}-\frac{1}{4 s}\right)}\right) .
\end{aligned}
$$

By substituting $\tau=s(\sin \theta)^{2}$ we have that

$$
\begin{aligned}
\int_{0}^{t} \mathrm{~d} \tau \tau^{-1 / 2}(s-\tau)^{-1 / 2}\left(1-\mathrm{e}^{-|x-w|^{2}\left(\frac{1}{4 \tau}-\frac{1}{4 s}\right)}\right) & \leqslant 2 \int_{0}^{\pi / 2} \mathrm{~d} \theta\left(1-\mathrm{e}^{-\frac{|x-w|^{2}(\cos \theta)^{2}}{4 s(\sin \theta)^{2}}}\right) \leqslant 2 \int_{0}^{\infty} \mathrm{d} \theta\left(1-\mathrm{e}^{-\frac{(|x|+R)^{2}}{s \theta^{2}}}\right) \\
& \leqslant(4 \pi)^{1 / 2}(|x|+R) s^{-1 / 2} .
\end{aligned}
$$

Then (152) is bounded from above by

$$
(4 \pi)^{2} C(K)(|x|+R) \int \mu_{K}(\mathrm{~d} y) \int \mu_{K}(\mathrm{~d} w) \int_{0}^{t} \mathrm{~d} s p(x, w ; s) p(x, y ; t-s) .
$$

But (154) has been estimated in (144). This completes the proof of (119), Lemma 9 and Proposition 2 for $m=3$.

Finally one can show that, by going through the estimates leading to the proof of Proposition 2, the remainder $\mathrm{O}\left(t^{-m / 2}\right)$ in Theorem 1 is uniform on compact subsets of $\mathbb{R}^{m} \backslash K$. This completes the proof of Theorem 1.

\section{Acknowledgements}

It is a pleasure to thank Erwin Bolthausen and Brian Davies for valuable discussions.

\section{References}

[1] M. van den Berg, Asymptotics of the heat exchange, J. Funct. Anal. 206 (2004) 379-390.

[2] M. van den Berg, On the expected volume of intersection of independent Wiener sausages and the asymptotic behaviour of some related integrals, J. Funct. Anal. 222 (2005) 114-128.

[3] M. van den Berg, On the volume of intersection of three independent Wiener sausages, in preparation.

[4] J.-F. Le Gall, Sur une conjecture de M. Kac, Probab. Theory Relat. Fields 78 (1988) 389-402.

[5] J.-F. Le Gall, Wiener sauasage and self-intersection local times, J. Funct. Anal. 88 (1990) 299-341.

[6] J.-F. Le Gall, Some properties of planar Brownian motion, in: École d'Été de Probabilités de Saint-Flour XX 1990, in: Lecture Notes in Mathematics, vol. 1527, Springer, Berlin, 1992, pp. 111-235.

[7] A. Joffe, Sojourn time for stable processes, Thesis, Cornell University, 1959.

[8] S.C. Port, Hitting times for transient stable processes, Pacific J. Math. 21 (1967) 161-165.

[9] S.C. Port, Hitting times and potentials for recurrent stable processes, J. Anal. Math. 20 (1967) 371-395.

[10] S.C. Port, C.J. Stone, Brownian Motion and Classical Potential Theory, Academic Press, New York, 1978.

[11] S.C. Port, Asymptotic expansions for the expected volume of a stable sausage, Ann. Probab. 18 (1990) $492-523$.

[12] F. Spitzer, Electrostatic capacity, heat flow and Brownian motion, Z. Wahrsch. Verw. Geb. 3 (1964) 110-121.

[13] A.-S. Sznitman, Brownian Motion, Obstacles and Random Media, Springer-Verlag, Berlin, 1998. 